\title{
Palm Vein Technology for Biometrics
}

\author{
Charu Jain, Ved Mishra, Aarti Chugh
}

\begin{abstract}
The use and implementation of biometrics for identification and authentication has become more important in the past decade. This is because there has been an increased risk associated with textual passwords such as dictionary attacks, eavesdropping, shoulder surfing etc. We have worked on Palm vein recognition for detecting palm veins in applications for biometric security using near infrared absorption phenomena. The goal is to produce a software prototype that is capable of identifying a person by the vein structures of the hand. The images used for the same were taken from the CASIA-MS-PalmprintV1 database collected by the Chinese Academy of Sciences' Institute of Automation (CASIA). After pre-processing, LDR and DCT have been used for feature extraction and Euclidian Distance is calculated for generating matching score. Acceptance/rejection is based on this matching score. The efficiency obtained was $93.2 \%$ when compared with other systems.
\end{abstract}

Index Terms: Biometrics, Near-Infrared Image, Palm Vein Recognition, Region of Interest.

\section{INTRODUCTION}

Biometric systems are authentication methods based on calculable physical or behavioral attributes that can be tested automatically. It is used as an alternative technique to textual passwords as it is more secure because textual passwords are subject to attacks which may lead to the loss of sensitive data. Therefore, biometrics has been introduced as an alternative technique to authenticate and validate a user. As automation is gaining popularity in each domain, similarly there is a huge demand of automated human authentication and identification biometrics for many applications as banks, companies etc. Palm vein technology has been proposed as human veins are unique for every individual and are difficult to copy. Human palms can be easily captured through scanning devices as images which can provide a variety of information $[1,5]$.

For vein pattern identification system, an infrared light source is used to scan hemoglobin within the

Revised Manuscript Received on June 15, 2019.

Dr. Charu Jain, Amity School of Engineering and Technology, Amity University, Gurgaon, India.

Dr. Ved Mishra, Amity University,Dubai

Aarti Chugh, Amity School of Engineering and Technology,

Amity University, Gurgaon, India.. blood. This is done by keeping a user's hand over a sensing device which can emit a near-infrared light. This light is then absorbed by the de-oxygenated blood that is flowing in the veins. This helps in extracting the vein structure of a person's palm as these veins show up on the image map as black lines, while the rest part of hand structure shows up as white. Due to complex vascular patterns in backside of hands and palms, many distinct features can be extracted for building robust vein biometric pattern matching system.

A. Kumar et al,[2] proposed automated Personal Authentication Using Hand Vein Triangulation and knuckle shape. The system employs dorsal hand vein images obtained from the low-cost, near IR contactless imaging camera. The knuckle tips are used as key points for the image normalization and to extract the region of interest. The experiment has been performed on 100 users and equal error rate of $1.14 \%$ has been achieved. Aycan Yuksel et. al. [3] proposed Hand vein biometry based on geometry and appearance methods. In proposed approach, a novel hand vein database and a biometric technique based on the statistical processing of the hand vein patterns are used.

Erdem Yoruk, Bulent Sankur, [4] proposed Shape-based hand recognition. In a pre-processing stage of the algorithm, the silhouettes of hand images are registered to a fixed pose, which involves both rotation and translation of the hand and, separately, of the individual fingers. Nisha Charaya et. al. [5] has presented various techniques used for dorsal hand vein authentication system. The authors also focused on methods used for feature extraction.

\section{METHODOLOGY}

Fig. 1 shows important steps involved in proposed vein pattern identification system. Development of palm vein biometric system starts with acquisition of human palm images through infrared emitting sensing device. After storing multiple images in computer database, pre-processing phase begins. This phase mainly involve extracting region of interest, binarisation etc. Next step focuses on feature extraction and finally feature matching. 


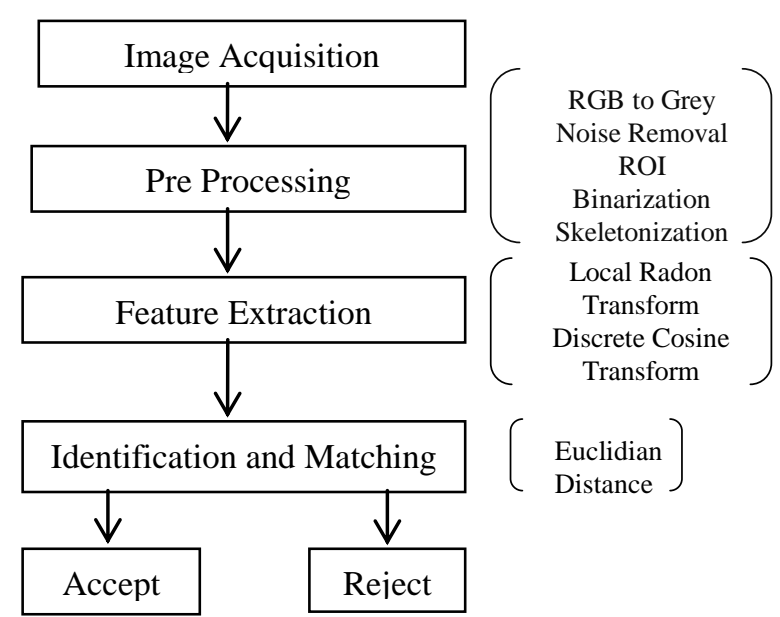

Fig.1: Block Diagram for Palm Vein Authentication System

\section{IMAGE ACQUISITION}

A multiple spectral imaging device is used to capture 7200 palm images from 100 individuals. The acquired scanned images are stored as 8 bit grayscale jpeg files. The database has been created in two different sessions. The interval between both the sessions is approximately 6 months. From this standard database, we have taken 30 images for training and identification. Fig. 2 shows sample images of palm vein. Every individual placed their palm into device where illumination was evenly distributed to have uniform background [2].

Fig. 3 shows Multi-Spectral Imaging Device developed by CASIA.

\section{PRE-PROCESSING}
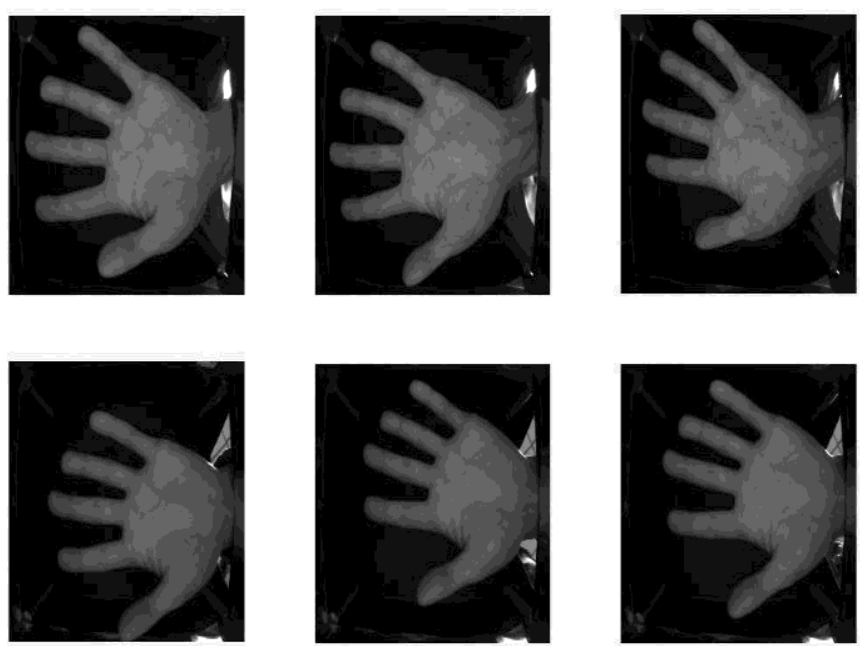

In this step noise removal and segmentation processes are performed on ROI in order to extract the palm vein pattern of an individual.

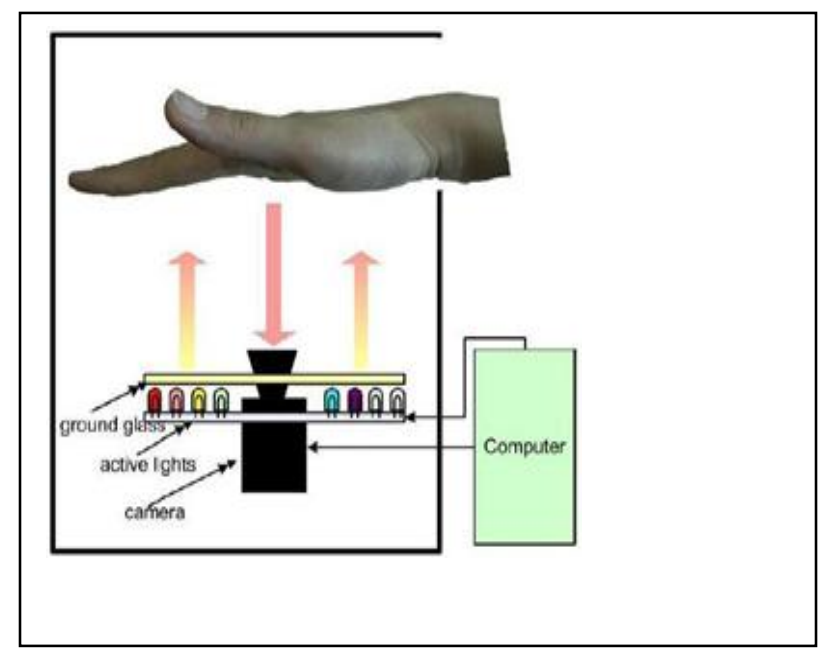

Fig.2: Sample Images of Palm

Fig.2: Sample Images of Palm

Fig.3: Multi-Spectral Imaging Device developed by CASIA

\section{A. Region of Interest Selection}

To increase the recognition accuracy and reliability, it is important to extract the features of vein patterns from the same region within different palm vein images. This process is known as extraction of region of interest. This option lets you extract the region of interest of an image. Extracted ROI is saved for further application of image pre-processing steps. Here, the database is populated by extracting the ROIs of all selected the samples.

\section{a. Interest Point}

Interest point shown in fig. 4 is some points in the hand that have specific interest maximum point on the contour. Which the maximum is set to the 3 highest contours. The three points are the point of the arch between the fingers. Those points located between pinkie and ring finger $(P 1)$, ring finger and middle finger $(P 2)$ and also between the middle finger and index finger $(P 3)$. 


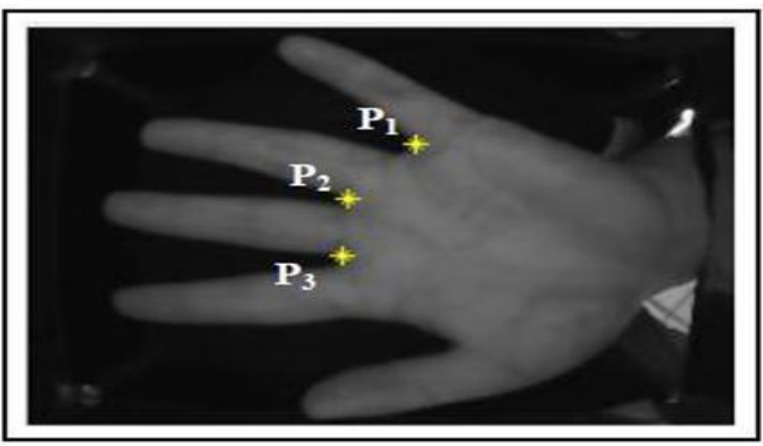

Fig.4: Interest Point

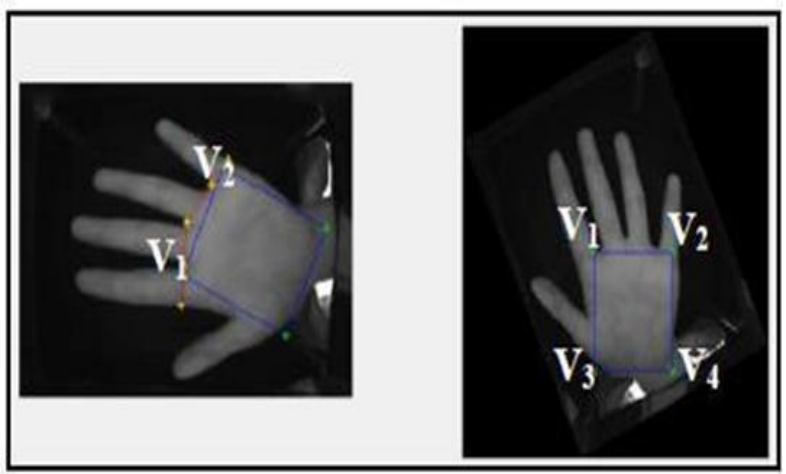

Fig.5: Final Region Selection

Next step is to pull out line from P1 to the furthest edge and set the middle point in between (V1). The second line is to pull out from P3 to the furthest edge in index finger area and also set the middle point in between (V2). Then, the points V1 and V2 showed in figure can be located. With the points V1 and V2, the ROI is defined as a rectangular region $\mathrm{V} 1-\mathrm{V} 2-\mathrm{V} 3-\mathrm{V} 4$, where $1 \mathrm{~V} 1 \mathrm{~V} 3=1.25 \times 1 \mathrm{~V} 1 \mathrm{~V} 2$. The image with ROI detected is rotated then so that line V1V2 is horizontal. The rectangular region is cropped and used for feature extraction to get the texture of vein. Fig. 5 shows final region selection.

\section{B. Noise Removal}

We have first used a median filter to remove the noise and then an anisotropic diffusion filter (ADF) for further filtering of the image. The filters were chosen as they preserve the edges while removing noise. Anisotropic diffusion is generally used in edge detection algorithms. Such algorithms run an edge seeking diffusion coefficient iteratively which breaks image piecewise. Each piecewise image constant has the boundaries between the constant components being detected as edges [3].Anisotropic Diffusion makes image
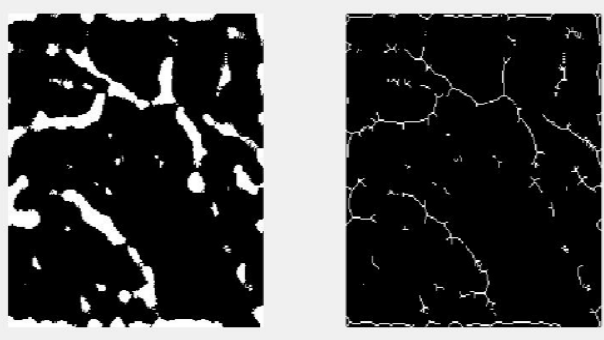

more smooth so in the next step edge can be detected more specifically.

\section{a. Median Filter}

Median filtering is a 2D filter, and works for grayscale images median filter is a non-linear operator. It replaces the pixel value in an image with the median value of its neighbors including itself. In fig 6. Median filter has been used to remove overall noise filtering.
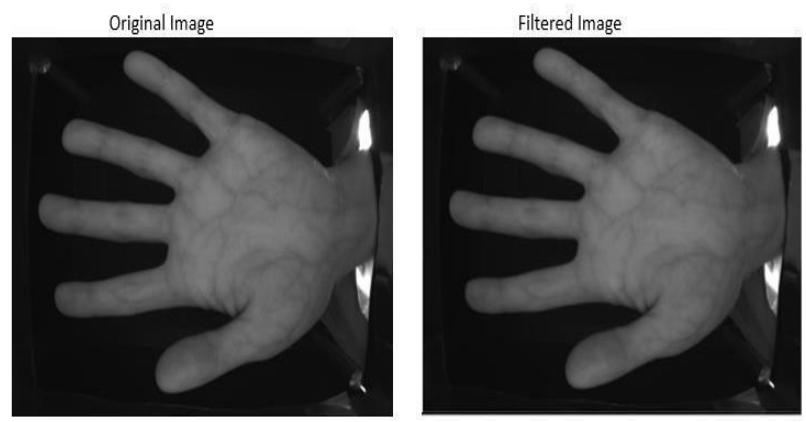

Fig.6: Results of Median Filter for Noise Removal

\section{spic Filter}

Anısotropic filtering (output shown in Fig. 7) is a method of enhancing the image quality of objects while preserving the edges of the objects. It eliminates aliasing effects, and at the same time reduces blur and preserves details in sub regions.
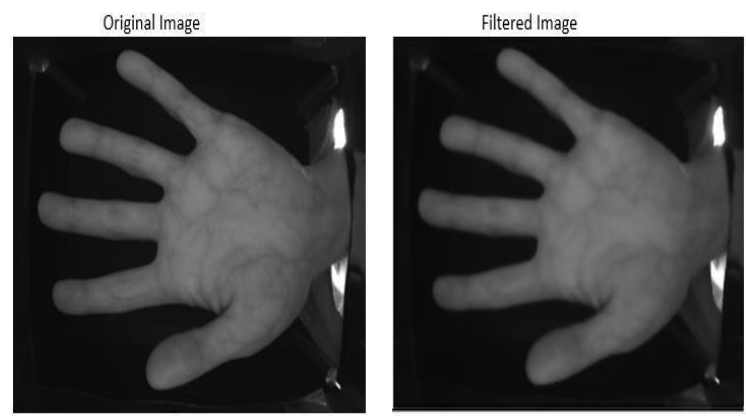

Fig.7: Output of Anisotropic Filtering

\section{Skeletonization}

Skeletonization is the process for reducing foreground region in a binary image to a skeleton remnant that largely preserves the extent and connectivity of the original region while throwing away most of the original foreground pixels. The width of the vein of an image varies with time in a person, therefore it is better to save the image skeleton for matching. These can be used as templates instead of binary image as it occupies lesser space (Fig. 9). 


\section{E. Contrast Enhancement}

Contrast Enhancement techniques have been widely used in many applications of image processing where the subjective quality of image is important for human interpretation. We have gone through various contrast enhancement techniques.

Adjust Method (Fig.10) maps the intensity value in Gray scale image to new value such that $10 \%$ of data is saturated at low level and $10 \%$ of data is saturated at high intensity of the image.
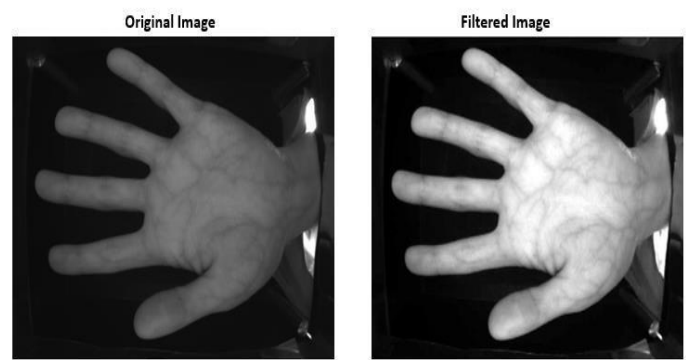

Fig.10: Output of Adjust Method of Contrast Enhancement

\section{F. Contrast-limited adaptive histogram equalization (CLAHE)}

CLAHE operates on small regions in the image, called tiles, rather than the entire image. Each tile's contrast is enhanced, so that the histogram of the output region approximately matches the histogram specified by the 'Distribution' parameter. The neighboring tiles are then combined using bilinear interpolation to eliminate artificially induced boundaries. The contrast, especially in homogeneous areas, can be limited to avoid amplifying any noise that might be present in the image (Fig. 11).
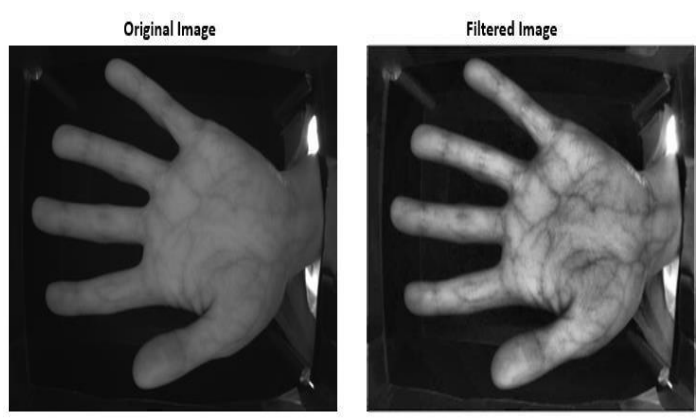

Fig.11: Output of CLAHE

\section{FEATURE EXTRACTION}

Feature extraction method is used to extract features to reduce the amount of redundant data for further analysis. Radon Transform is used to define the local features and it basically represents the texture information of palm vein image. In 2-d space, it can be represented as (1).

$\mathrm{RT}(\mathrm{x}, \theta)=\int \mathrm{f}\left(\mathrm{y}_{1}, \mathrm{y}_{2}\right) \quad \delta \quad\left(\mathrm{y}_{1} \cos \theta+\mathrm{y}_{2} \sin \theta-\mathrm{x}\right) \quad \mathrm{dy}_{1} \mathrm{dy}_{2}$

Where $\delta$ represents the Dirac distribution. Radon transform calculates the line integral along with parallel paths in various different directions. It is applied on palm vein image to obtain the projection of intensities at different angles. It maps Cartesian rectangular coordinates with respect to distance and angles. 60 different angles are computed in Radon space to enhance low frequency components. Discrete Cosine Transform (DCT) is then applied to obtain frequency features in Radon space [13]. 25\% of DCT coefficients are summed to form feature vector. These coefficients are calculated in order to reduce the dimensionality of the feature vector.

\section{IDENTIFICATION AND MATCHING}

Feature vector obtained is now stored in database as a reference feature vector for identification. Whenever the test image is fed to the system for identification, feature vector obtained from test image is compared with the reference feature vector stored in the database. Based on the match score generated, the decision of acceptance or rejection is made. The match score is generated on the basis of Euclidian distance.

\section{CONCLUSION}

Vein pattern recognition has been selected as it is almost impossible to forge a fake vein pattern as the veins lie deep within the skin of the palm whereas, due to the long legacy of fingerprints, there are a number of methods that have been created to fool fingerprint systems. The proposed system has been implemented and tested with Chinese Academy of Sciences 'Institute of Automation(CASIA). Data base consists of 7200 palm images of 100 individuals using specialized scanner. Out of which 30 samples were selected randomly in 2 different sessions. The time interval between both the sessions was approximately six months. Radon Transform and DCT were used to extract features and matching was done on the basis of Euclidian distance. The efficiency obtained was 93.2\%. Results indicate that palm vein technology is one of the promising form of biometric identification.

\section{REFERENCES}

1. Mr. Vishal U. Bhosale, Mr. Onkar S. Kale, Mr. Mahesh W. Pawar, Mr. Roshan R. Patil, Mr. Pritam S. Patil, Prof Mrs. Sonali Madankar," Palm Vein Extraction and Matching For Personal Identification", IOSR Journal of Computer Engineering (IOSR-JCE) e-ISSN: 2278-0661, p- ISSN: 
2278-8727 Volume 16, Issue 2, Ver. IX (Mar-Apr. 2014), PP 64-68.

2. Ajay Kumar, et al., "Personal Authentication Using Hand Vein Triangulation and Knuckle Shape.", IEEE Transaction on Image Processing. Vol.18, no.9, September 2009, pp21272136.

3. Aycan Yuksel, Lale Akarun, Bulent Sankur, Jie Cao, "My Palm Vein: A Palm Vein-based Lowcost Mobile Identification System For Wide Age Range", 17th International Conference on E-health Networking Application \& Services 2015.

4. N. Miura, A.Nagasaka, T. Miyatake, "Feature extraction of finger-vein patterns based on repeated line tracking and its application to Person Identification", Machine Vision and Applications, vol.15, no.4, 2004, pp.194-203.

5. Nisha Charaya, Dr. Priti Singh"International Journal of Pure and Applied Mathematics", Volume 119, No. 16, 2018, pp 2175-2185.

6. Jason Forté,'Development of a Near Infrared Hand Vein Imaging Device With Software Enhancement", Presented at University of Cape Town, November 2014.

7. Jayanti Yusmah Sari, Chastine Fatichah, and Nanik Suciati "Local Line Binary Pattern For Feature Extraction On Palm Vein Recognition", Department of Informatics Engineering, Faculty of Information Technology, Institute Teknologi Sepuluh Nopember Surabaya Keputih, Sukolilo, Surabaya 60111, East Java, Indonesia

8. Yingbo Zhou and Ajay Kumar, "Human Identification Using Palm-Vein Images", IEEE Transaction on Information Forensics and Security, December 2011, VOL.6, NO.4

9. Sheetal, Ravi Prakash Goel, Kanwal Garg "Image Processing in Hand Vein Pattern Recognition System”, Volume 4, Issue 6, June 2014, International Journal of Advanced Research in Computer Science and Software Engineering

10. Dattatray V. Jadhav, Raghunath S. Holambe "Radon and discrete cosine transforms based feature extraction and dimensionality reduction approach for face recognition", Department of Instrumentation, SGGSIE \& T, Vishnupuri, Nanded (MS), Maharashtra 411037, India

11. Vijayta Chowdhary, Kamini Verma, Himanshu Monga "Human Identification Using Palm-Vein Images Using Gabor Filter", Volume 4, Issue 7, July 2014 ISSN: 2277 128X, International Journal of Advanced Research in Computer Science and Software Engineering

12. Anisotropic Diffusion, "http://www.mathworks.com/ matlabcentral/fileexchange/14995-anisotropic-diffusion--per ona---malik-/content/anisodiff_PeronaMalik/ anisodiff2D.m.

13. Sudha Yadav, Charu Jain, Aarti Chugh, "Evaluation of Image Deblurring Techniques” IJCA, vol. 139, 2016.

14. Charu Jain, Aarti Chugh, Dr. Priti Singh, "An Offline Signature verification using Adaptive Resonance Theory” IJCA, vol 94, issue 2, 2014, pp 8-11.

\section{AUTHORS PROFILE}

Dr. Charu Jain has completed her PhDin Artificial Intelligence and has published more than 30 research papers in reputed National/International Journals and Conferences. She has authored 4 books in her field.

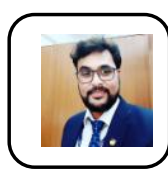
Amity University Dubai as Head of Academics-Engineering. He has published more than 20 research papers and authored one book of his field. His major interests of areas are Network Security, Data Mining and Process Mining.

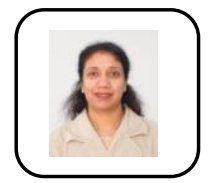

Aarti Chugh is motivated teaching professional $(15+$ experience) with zeal for gaining knowledge about new technologies. She has published more than 20 research papers in reputed National/International Journals and is working on projects based on Artificial Intelligence and Big Data. 